Research Paper

\title{
Patient Satisfaction with Physician Discussions of Treatment Impact on Fertility, Menopause and Sexual Health among Pre-menopausal Women with Cancer
}

\author{
Maura Scanlon ${ }^{1}$, Anne Blaes$^{2}$, Melissa Geller ${ }^{3}$, Navneet S Majhail2 ${ }^{2}$ Bruce Lindgren ${ }^{4}$, Tufia Haddad $^{2} \bowtie$ \\ 1. University of Minnesota Medical School, Minneapolis, MN; \\ 2. University of Minnesota, Department of Medicine, Division of Hematology, Oncology and Transplantation; \\ 3. University of Minnesota, Department of Obstetrics, Gynecology, and Women's Health; \\ 4. University of Minnesota Masonic Cancer Center, Biostatistics and Bioinformatics Core.
}

Corresponding author: Tufia Haddad, MD. Mayo Clinic, Department of Oncology, 200 First Street SW, Rochester, MN 55905, Fax: 507-284-1803.

(c) Ivyspring International Publisher. This is an open-access article distributed under the terms of the Creative Commons License (http://creativecommons.org/ licenses/by-nc-nd/3.0/). Reproduction is permitted for personal, noncommercial use, provided that the article is in whole, unmodified, and properly cited.

Received: 2012.03.27; Accepted: 2012.05.08; Published: 2012.05.16

\begin{abstract}
PURPOSE: Pre-menopausal women with cancer are at risk of therapy-associated infertility, premature menopause, and sexual dysfunction. However, it is unknown whether oncologists adequately address these risks during treatment planning. We conducted a study to evaluate physician-patient discussions addressing the impact of cancer treatment and actual treatment effects on fertility, menopause status, and general sexual health.

METHODS: A questionnaire was administered in four oncology clinics specializing in breast, gynecologic, general hematology-oncology, and blood and marrow transplantation (BMT) cancer care at a single institution. Eligible participants were pre-menopausal at the time of diagnosis and either actively receiving or within 24 months from completion of treatment. Participants completed the questionnaire at enrollment and at I-year follow-up.

RESULTS: Of the 104 eligible women, a majority were satisfied with the quality (68\%) and length (66\%) of reproductive health discussions, with the highest satisfaction levels in the gynecologic cancer clinic (85\%) and the lowest levels in the BMT clinic (53\%). Fertility preservation was desired by $20 \%$ of women, including some $>40$ years old. Women were more interested in discussing treatment impact on menopause status and sexual health than fertility. Rates of discussions on treatment impact on sexual health were low despite $77 \%$ of women reporting severe sexual dysfunction at I-year follow-up.

CONCLUSIONS: One-third of women are dissatisfied with the quality and length of discussions regarding the impact of cancer treatment on reproductive health. There is notably inadequate counseling on the effect of treatment on fertility in women $>40$ and on sexual function in all women. Oncologists must offer better resources and improve communication on the effect of treatment on reproductive health to pre-menopausal women with cancer.
\end{abstract}

Key words: Cancer, Fertility, Physician-Patient Discussions, Women, Sexual Health.

\section{Introduction}

As the population of cancer survivors continues to grow, it is becoming increasingly imperative for oncologists to identify and address important quality-of-life issues that negatively affect the well-being of 
their patients. Over $60 \%$ of cancer survivors in the United States are women. ${ }^{1}$ Quality-of-life concerns for these cancer survivors create new challenges for oncologists to address during treatment planning and long-term follow-up. Recent studies show that young women with cancer have concerns related to sexual health, treatment-induced infertility, and menopause. ${ }^{2-4}$ While the importance of these quality-of-life issues is ill-defined, it is clear that these common side effects of cancer treatment occur.

Chemotherapy may reduce the number of viable ovarian follicles in a drug and dose-dependent response, and surgical or radiation treatment may induce changes in uterine, fallopian, or ovarian anatomy which interferes with a woman's ability to conceive after cancer therapy is completed. ${ }^{5}$ Young women experiencing chemotherapy or surgically-induced amenorrhea are likely to exhibit menopausal symptoms, including hot flashes, insomnia, and fatigue. ${ }^{6}$ Chemotherapy, surgery, radiation, or endocrine treatment may also result in sexual dysfunction, such as loss of libido, vaginal dryness, dyspareunia, and decreased personal and partner satisfaction. 7,8 Sexual dysfunction affects up to $90 \%$ of women treated for breast cancer, with vaginal dryness being the most significant indicator of long-term problems in sexual functioning. ${ }^{7-12}$ Many other factors may contribute to chronic sexual dysfunction including the psychological effects of the cancer diagnosis, anxiety of recurrence, depression, and impaired body image. Physician-patient counseling about the impact of cancer treatment on general reproductive health should be an integral part of the patient care experience for pre-menopausal women with cancer.

Recent surveys of cancer survivors of reproductive age show that a majority have no recollection of discussing fertility at the time of treatment planning. ${ }^{13}$ The American Society of Clinical Oncology (ASCO) has recognized the need for improvement in physician-patient counseling on these topics. ASCO has created specific recommendations to guide physicians in discussing with patients the possibility of infertility due to cancer therapy and offering referrals to reproductive specialist care. Over half of physicians are not following these recommendations for reproductive-age patients in terms of offering fertility specialist care to those patients who are interested in preserving fertility and at risk for treatment-induced infertility. ${ }^{13}$ Few data exists on the personal importance of such discussions on fertility and sexual health for women facing a new cancer diagnosis. The purpose of the present study was to determine whether fertility, menopause status, and sexual health were important quality-of-life concerns among pre-menopausal women with cancer and whether oncologists discussed these concerns adequately during treatment planning and long-term follow-up.

\section{Methods}

\section{Patients}

All subjects were recruited from the University of Minnesota Cancer Clinics by study investigator, M.S., who had no direct involvement in their clinical care. The subjects were either receiving care in the general hematology-oncology clinic or in one of three sub-specialty clinics focused on breast, gynecologic, or blood and marrow transplantation (BMT) cancer care. These four clinics were staffed with designated and non-overlapping oncologists. Informed consent was obtained by investigator M.S. in the clinic prior to screening patients for eligibility. Women were eligible to participate in this study if they had a histologically proven diagnosis of cancer, were 18 years of age or older, and were actively receiving cancer therapy or within 24 months from the end of their cancer treatment. All subjects were pre- or peri-menopausal at the time of their cancer diagnosis, as defined by the presence of at least two menstrual periods within 6 months of diagnosis. Subjects were excluded if they were post-menopausal at diagnosis or had metastatic disease.

\section{Study design}

We conducted a longitudinal cohort study of all eligible female cancer patients receiving treatment at our institution. Participants were asked to complete an original 24-item questionnaire (see Supplementary Material) designed to assess discussions they had with their treating oncologist. We evaluated patient satisfaction levels of the physician-patient discussions regarding treatment impact on fertility, menopause status, and sexual health, as well as the personal importance of having such discussions on these topics. The questionnaire was divided into three sections to assess these discussions throughout the spectrum of cancer care, to include: before cancer diagnosis, during treatment planning, and at the time of study enrollment. A follow-up survey on these topics was administered by mail one year after enrollment and it was comprised of repeated sections from the original questionnaire concerning the overall discussion satisfaction ratings and treatment impact on sexual health. A second mailing with the follow-up survey was sent to those patients who did not respond to the original request. Questionnaire data was linked with information extracted from the medical record, including date of birth, age at diagnosis, menopausal 
status at the time of diagnosis, tumor histology, stage at diagnosis, and all cancer treatment received. The protocol and analysis were approved by the University of Minnesota Institutional Review Board. All patients provided written informed consent according to the Declaration of Helsinki.

\section{Statistics}

Each questionnaire item was summarized by the number and percent for each response. The percentages were based on the number of women who answered the question, unless that question was not applicable (N/A), in which case the subject was not counted. Items on the Likert scale of 1 (very negative) to 5 (very positive) were reduced into three categories $(1+2,3,4+5)$ for reporting purposes. However, for comparisons between patient groups - such as disease type, age groups and gender of oncologist - the original 5-level ordinal scale was analyzed by either the non-parametric Kruskal-Wallis test or Wilcoxon rank sum test. Other questionnaire items were evaluated between groups using Fisher's exact test. Infrequent responses such as "Unsure" or "Cannot recall" were excluded from statistical comparisons to improve interpretation of results. Changes in women's responses between the initial questionnaire and the same questions asked on the follow-up questionnaire were evaluated by McNemar's chi-square test. For these before-and-after comparisons, just two Likert subcategories $(1+2+3$ and $4+5)$ were used. All statistical analyses were performed using SAS version 9.2 (SAS Institute Inc., USA). A p-value of $<0.05$ was considered statistically significant.

\section{Results}

\section{Patient characteristics}

One hundred and twenty-six women were screened, with 114 consenting to participate. Ten women were excluded upon final eligibility review due to the lack of malignancy $(n=4)$ and post-menopausal status at cancer diagnosis $(n=6)$, resulting in 104 evaluable subjects divided into four disease-specific cohorts (Table 1). Enrollment in the study occurred at a median time of 2.4 years after cancer diagnosis. Gender of the treating oncologist was approximately evenly distributed between males $(47 \%)$ and females (52\%). Median age at study enrollment was 40.5 years (range, 18-52 years). Most of the participants were either married or single and in a relationship (77\%). Sixty-seven $(64 \%)$ women experienced treatment-induced amenorrhea, as defined by no menses for greater than 12 months. All women with gynecologic malignancy and some with breast cancer experienced surgically-induced menopause as a result of treatment $(n=37)$. Of the 67 women who did not undergo surgically-induced menopause, 38 (56\%) went on to experience amenorrhea.

Table I: Demographics and clinical characteristics of subjects.

\begin{tabular}{|c|c|c|c|c|c|}
\hline Disease & All & Breast & Gyn & General & BMT \\
\hline $\mathrm{N}(\%)$ & $104(100)$ & $38(36)$ & $26(25)$ & $20(19)$ & $20(19)$ \\
\hline One year follow-up & $53(100)$ & $21(40)$ & $14(26)$ & $9(17)$ & $9(17)$ \\
\hline \multicolumn{6}{|l|}{ Age } \\
\hline$<35$ & $27(26)$ & $5(13)$ & $4(15)$ & $7(35)$ & $11(55)$ \\
\hline $35-39$ & $21(20)$ & $9(24)$ & $7(27)$ & $3(15)$ & $2(10)$ \\
\hline $40-44$ & $28(27)$ & $9(24)$ & $9(35)$ & $6(30)$ & $4(20)$ \\
\hline$>44$ & $28(27)$ & $15(39)$ & $6(23)$ & $4(20)$ & $3(15)$ \\
\hline \multicolumn{6}{|l|}{ Marital Status } \\
\hline Single & $41(40)$ & $13(34)$ & $13(50)$ & $9(45)$ & $6(32)$ \\
\hline Married & $60(58)$ & $24(63)$ & $13(50)$ & $10(50)$ & $13(68)$ \\
\hline Divorced & $2(2.0)$ & $1(3.0)$ & $0(0.0)$ & $1(5.0)$ & $0(0.0)$ \\
\hline Median Time Since Diagnosis (yrs) & 2.4 & 2.8 & 1.5 & 2.2 & 3.0 \\
\hline \multicolumn{6}{|l|}{ Gender of Oncologist } \\
\hline Male & $46(47)$ & $18(51)$ & $3(12.5)$ & $11(61)$ & $14(70)$ \\
\hline Female & $50(52)$ & $17(49)$ & $21(87.5)$ & $7(39)$ & $5(25)$ \\
\hline \multicolumn{6}{|l|}{ Treatment Received $^{a}$} \\
\hline Surgery & 73 & 36 & 25 & 12 & 0 \\
\hline Chemotherapy & 80 & 29 & 14 & 17 & 20 \\
\hline Radiation & 33 & 12 & 9 & 8 & 4 \\
\hline Hormone Therapy & 26 & 26 & 0 & 0 & 0 \\
\hline Stem Cell Transplant & 22 & 0 & 0 & 0 & 22 \\
\hline Treatment-induced Amenorrhea & $67(64)$ & $23(60)$ & $22(84)$ & $6(30)$ & $16(80)$ \\
\hline
\end{tabular}

a Individual subjects may have received more than one modality of cancer therapy. 
During the course of the year between enrollment and the 1 year follow-up survey, five patients died due to disease progression. Of the remaining 99 women, 53 completed the follow-up questionnaire for a response rate of $54 \%$. Of the patients who responded to the follow-up survey, $36 \%$ were under the age of 39 and $64 \%$ were ages 40 or older; whereas in the original survey cohort the distribution of patient age was $46 \%$ under the age of 39 and $54 \%$ ages 40 or older. Most women who completed the follow-up survey were married $(66 \%)$, similar to the percentage of married patients in the original survey (58\%); however non-responders to the follow-up survey were less likely to be married (48\%). There were no differences between the original and 1-year follow-up cohorts in terms of the distribution of patients by disease-specific cancer care (Table 1). Sixty-six percent of women in the follow-up survey were amenorrheic as compared to $64 \%$ in the initial survey. In general, the only appreciable difference between the patient characteristics of those who responded to the 1-year follow-up survey and those who completed the original survey is the distribution of patient age. Compared with the 1-year follow-up responders, non-responders were more likely to be younger (less than 40) and single.

\section{Patient Satisfaction with quality-of-life discus- sions during treatment planning}

Patient satisfaction was assessed during treatment planning in terms of the quality and length of physician-patient discussions about fertility, menopause, and sexual health topics (Figure 1). Overall, a majority of women were satisfied with the quality $(68 \%)$ and length $(66 \%)$ of discussions during treatment planning (Figure 1). Disease-specific cancer care significantly affected satisfaction ratings in the quality and length of conversation. Satisfaction was highest for the quality and length of conversation among gynecologic cancer patients and lowest among BMT cancer patients. Patients were most likely to be dissatisfied with conversation quality and length in the breast cohort, $(p=.012, p=.036$ respectively). Gender of the oncologist, patient age, and initial interest in fertility preservation did not significantly affect patient satisfaction ratings for either conversation quality or length. Initial satisfaction levels remained unchanged among those women who responded to the 1-year follow-up study $(\mathrm{n}=53)$.

\section{Time spent on reproductive and sexual health discussions}

Forty seven (62\%) women spent 1-10 minutes in their initial discussion of treatment impact on fertility, menopause, and sexual health with their treating oncologist while only 29 (38\%) women spent $>10$ minutes in such discussions. Disease-specific cancer care significantly affected the length of initial discussions $(p=.001)$. Discussions lasting more than 10 minutes occurred for $14(61 \%)$ women in the gynecologic clinic, $9(33 \%)$ women in the breast clinic, 5 (45\%) in the general oncology clinic, and only $1(7 \%)$ in the BMT clinic. Although 38 (41\%) women had two or more follow-up discussions about fertility, menopause, and sexual health, $25(27 \%)$ women reported no additional discussions. Fertility and reproductive health discussions were physician-initiated $73 \%$ of the time, whereas 19 (23\%) women had to initiate the conversation on their own. Gender of oncologist, disease-specific cancer care, or patient age did not significantly affect the number of follow-up discussions or the initiator of discussions.

\section{Fertility and sexual health resources}

Only $13(14 \%)$ women were encouraged to speak to a fertility specialist about fertility preservation. Age significantly affected this result, with $9(38 \%)$ women $<35$ years old and only $1(2 \%)$ woman $\geq 40$ years old receiving this recommendation $(\mathrm{P}=.001)$; gender of the oncologist or disease-specific cancer care did not affect the chance of fertility specialist referral. Additional resources on reproductive health topics were provided to $35(37 \%)$ women by their treating oncologist. Women in the gynecologic cancer clinic were 2-3 fold more likely than those in other clinics to receive these additional resources $(p=.026)$. Fifty-one $(54 \%)$ women reported searching for additional resources on their own.

\section{Patient attitudes about fertility preservation over time and their impact on cancer treat- ment choice}

Nineteen $(20 \%)$ women ranked fertility preservation as important at the time of diagnosis and treatment planning. These women were more likely to be of a younger age and with no previous pregnancies. While fertility preservation was ranked as important $16 \%$ of women $40-44$ years old, only one woman over the age of 40 was referred to a fertility specialist. Even though none of the women $>44$ years old were interested in preserving their own fertility, $12 \%$ of these women found it important to discuss the risk of treatment-induced infertility. In addition, the risk of infertility affected treatment choice in 12 (13\%) subjects; factors significantly affecting this result were younger age, being unmarried, and having no prior 
successful pregnancies.

After treatment completion, interest in fertility preservation was unchanged in $70 \%$ of women who were initially interested in fertility preservation and did not undergo surgically-induced menopause. Marital status and disease-specific cancer care did not affect the importance assigned to fertility preservation over time. Concern about cancer recurrence did affect interest in future pregnancies in $29 \%$ of women.

\section{Patient attitudes about reproductive and sexual health discussions}

Thirty-two (31\%) women reported that it was important to have a discussion about treatment impact on fertility at the time of diagnosis (Figure 2a). Women were more interested, however, in discussing treatment impact on menopause status $(n=69,68 \%$; Figure $2 b)$ and general sexual health $(n=55,55 \%$; Fig- ure 2c). In general, fewer women were interested in discussing the impact of treatment on fertility or menopause status $(33 \%, 68 \%$ respectively) than the percentage of those who actually had such a discussion with their oncologist $(77 \%, 82 \%$ respectively). In contrast, more women (55\%) were interested in discussing treatment impact on general sexual health than the percentage of those $(40 \%)$ who actually had such a discussion with their oncologist. This discrepancy was exacerbated by disease-specific cancer care cohorts. Only $22 \%$ of women in the gynecologic clinic reported no conversation of treatment impact on sexual health, whereas $80 \%$ of those in the breast cancer clinic and $82 \%$ in the BMT clinic reported no such discussion $(\mathrm{p}<.001)$. There was also significantly higher incidence of discussions of treatment impact on sexual health led by female oncologists $(p=.02)$.

\section{A Satisfaction with Quality of Discussion}

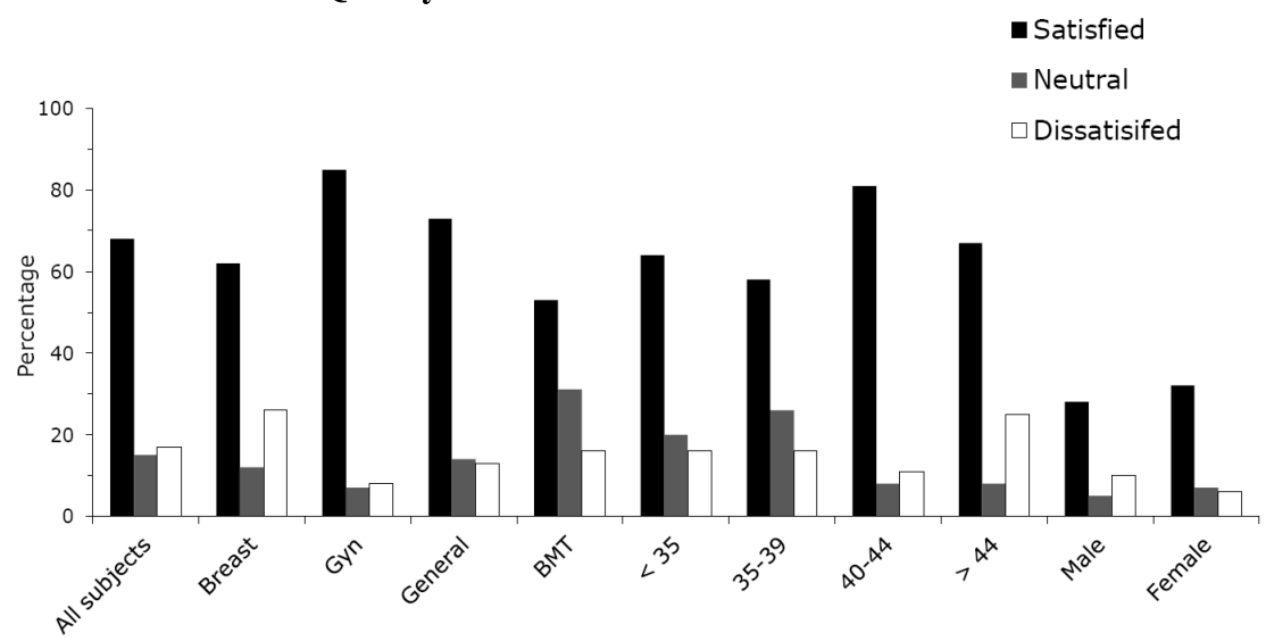

\section{B Satisfaction with Length of Discussion}

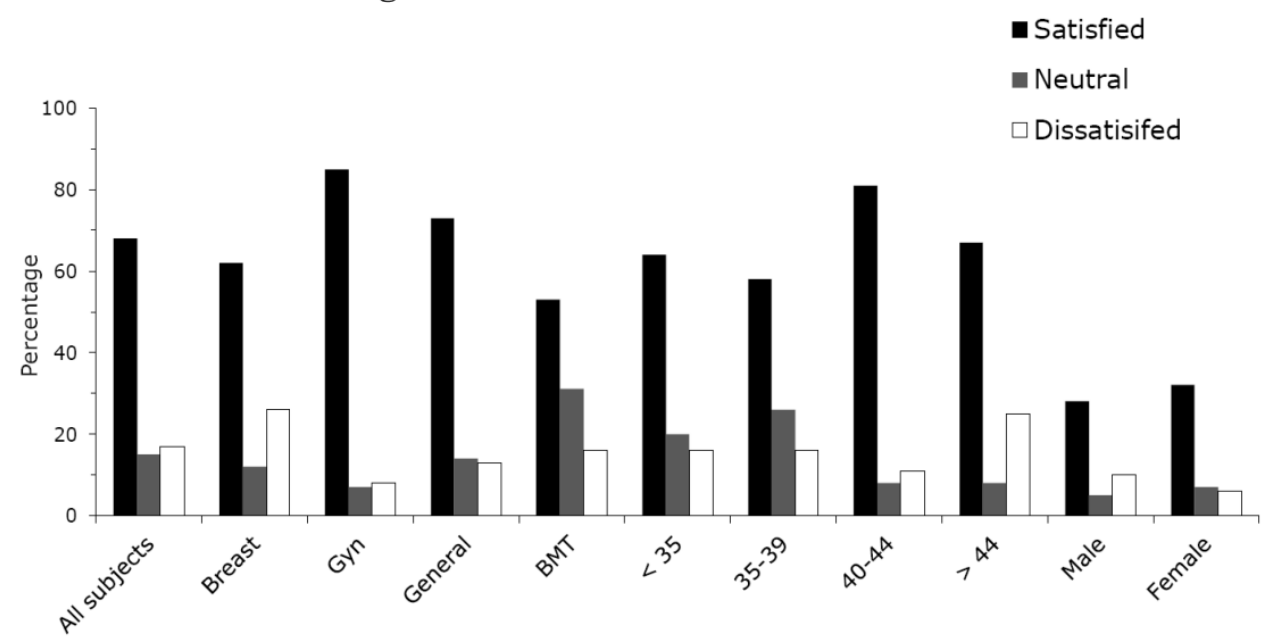

Fig I. Patient satisfaction with quality (A) and length (B) of physician-patient discussions on treatment impact on fertility and sexual health. A Satisfaction with Quality of Discussion. 


\section{A. Fertility}

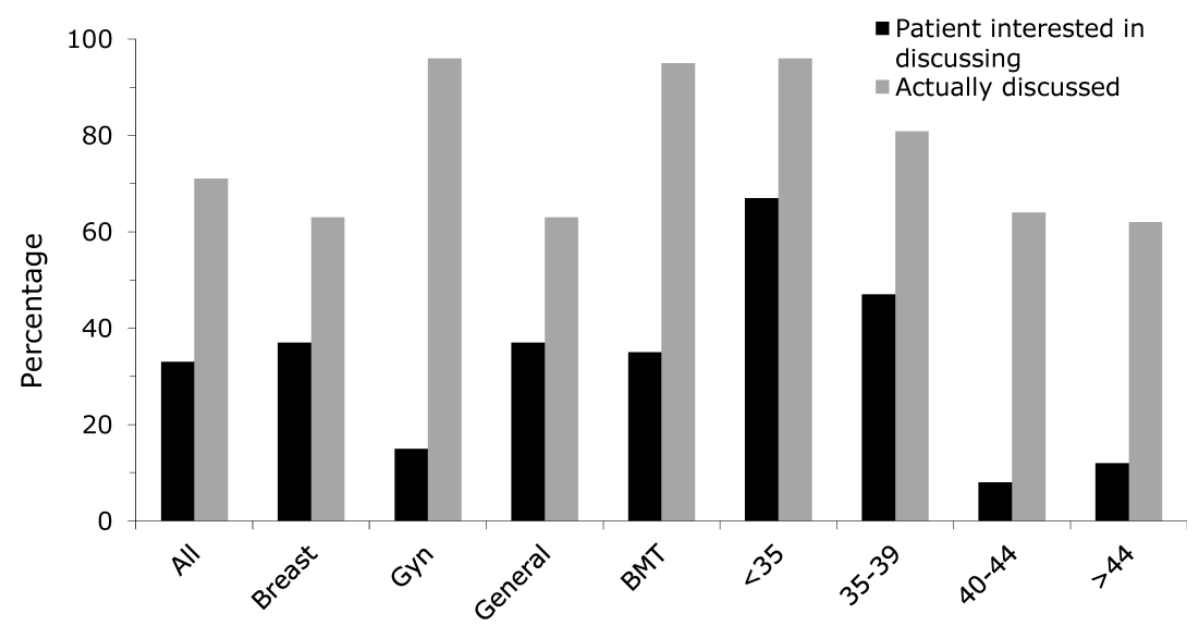

\section{B. Menopause}

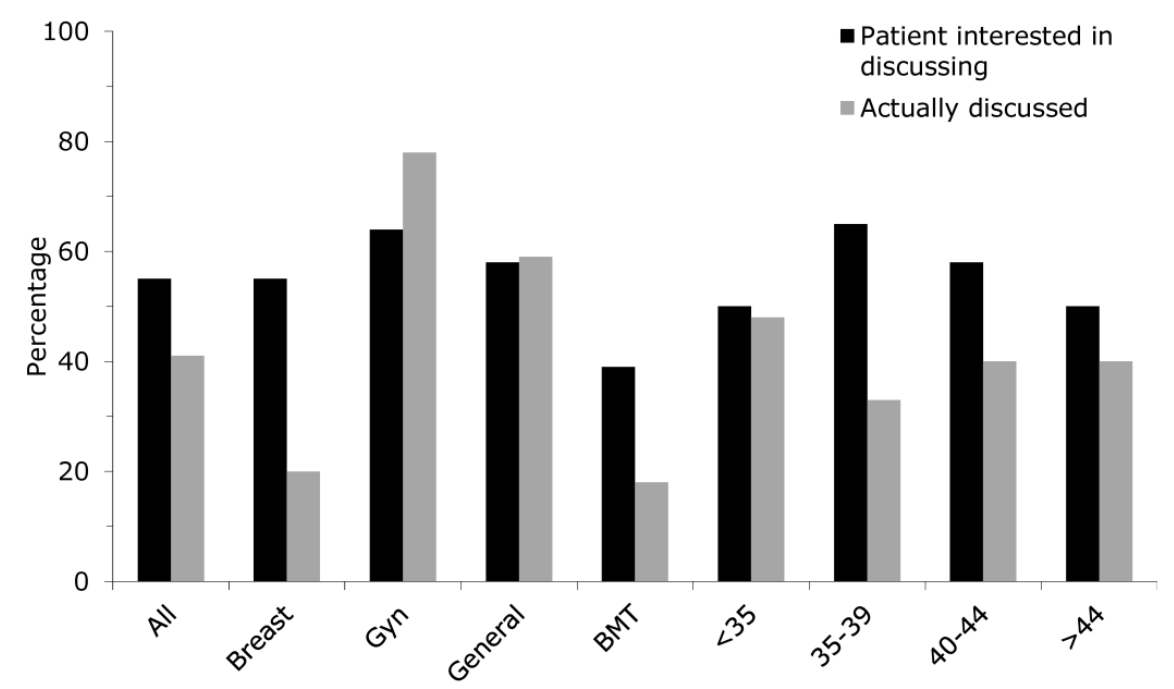

\section{Sexual Health}

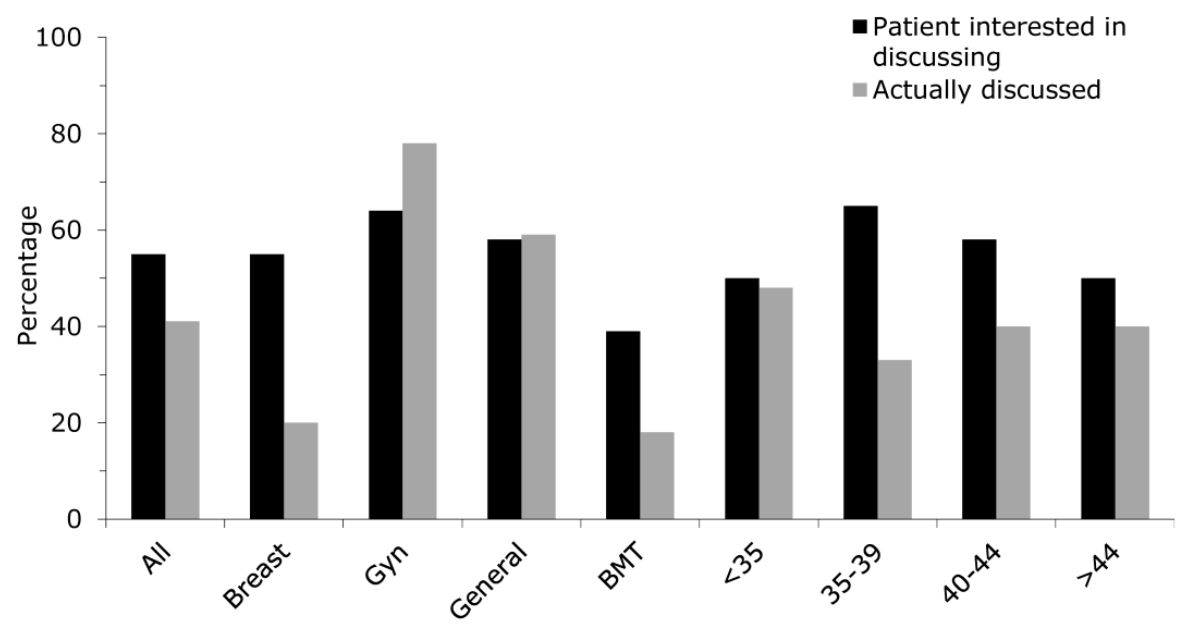

Fig 2. Patient interest and discussions that occurred regarding treatment impact on fertility (A), menopause status (B), and sexual health (C). 


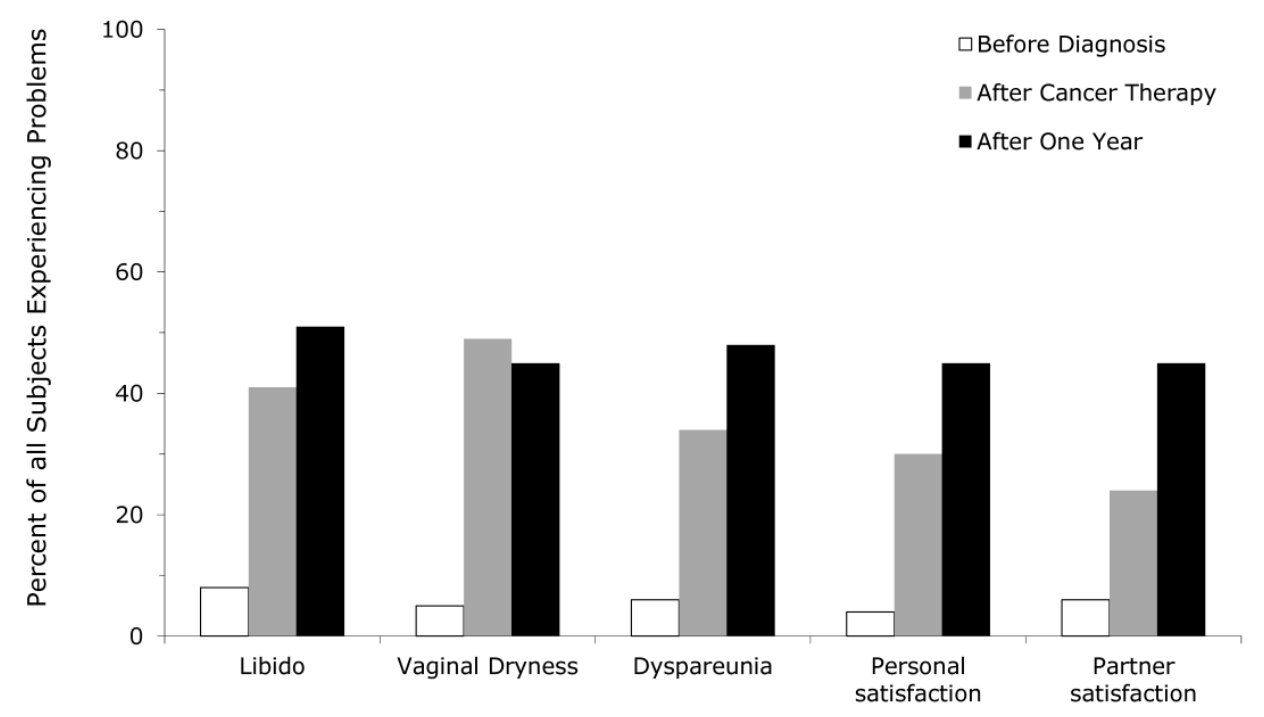

Fig 3. Changes in sexual dysfunction over time.

The importance of discussing treatment impact on general sexual health was rated significantly higher at 1-year follow-up. Thirty-two $(62 \%)$ women in the follow-up survey had initially ranked sexual health discussions as important compared with 41 $(79 \%)$ one year later $(p=.039)$. Age, marital status, and menopausal status did not affect attitudes toward reproductive or sexual health discussions. Patient attitudes about discussing treatment impact on fertility or menopause remained unchanged.

\section{Treatment impact on sexual health over time}

Rates of sexual dysfunction were high and increased over time, with $77 \%$ of women reporting severe problems with at least one of the sexual health domains (libido, vaginal dryness, dyspareunia, patient satisfaction and partner satisfaction) at 1-year follow-up (Figure 3). Dyspareunia $(p=.058)$ and partner satisfaction $(\mathrm{p}=.013)$ specifically were significantly worse at 1-year follow-up. Only menopausal status, but not age or marital status, significantly influenced rates of sexual dysfunction. At 1-year follow-up, 19 $(83 \%)$ women experiencing severe sexual side effects were amenorrheic, whereas only $4(17 \%)$ were not amenorrheic $(p=.027)$. The rate of amenorrhea was unchanged over time $(64 \%$ at baseline versus $66 \%$ at follow-up), therefore the higher rates of sexual dysfunction likely represent the effect of prolonged amenorrhea rather than higher rates of it.

\section{Patient interest in alternative methods of fer- tility after treatment}

After cancer treatment, there were 67 women who had not experienced surgically-induced meno- pause. Of these women, $21 \%$ reported interest in adoption, surrogacy, or other methods of fertility after treatment. Younger age and lack of previous pregnancies, but not disease-specific cancer care, were significantly associated with interest in adoption, surrogacy, and other methods of fertility.

\section{Discussion}

The present study demonstrates that female cancer survivors are generally satisfied with physician-patient discussions of treatment impact on fertility, menopause status and sexual health as they navigate through the cancer treatment process. Satisfaction rates were markedly different within the disease-specific clinics, with highest levels achieved in the gynecologic cancer clinic. These results are not unexpected, given the proximity of the cancer to reproductive organs, physician familiarity with these topics, and the fact that more time was allocated to discuss these topics in clinic. However, one area of concern raised by our study is that discussions of treatment impact on general sexual health were infrequent despite high demand for them. This is troubling since the overwhelming majority of women experienced severe sexual dysfunction, especially in those who also experienced treatment-induced amenorrhea. This finding is consistent with previous reports. 7,8 Additionally, we found that disturbances in sexual function were not short-lived and may have worsened over time. Oncologists, and in particular male oncologists, did not discuss the effect of treatment on general sexual health as frequently or as in-depth as patients had hoped. This may be explained by a general lack of knowledge and training 
on this topic, in addition to its sensitive nature. Taken together, these data demonstrate that the impact of cancer treatment on general sexual health is particularly important for oncologists to address during treatment planning and in survivorship follow-up.

Studies involving female cancer survivors indicate that many women desire to preserve fertility during cancer treatment. . $^{3,13-15}$ We found that the level of interest in fertility preservation persisted post-therapy and had the power to influence cancer treatment choice when women had options, particularly in the nulliparous cohort. Younger women were more likely to explore options such as surrogacy and adoption, further highlighting the necessity for access to fertility specialist expertise. The paucity of additional resources and fertility specialist referrals to supplement reproductive health discussions and to aid women in cancer treatment decisions is concerning and reflects national trends. Our data demonstrate there is a significant desire for knowledge and counseling of reproductive options at the time of cancer diagnosis regardless of age and initial interest in fertility preservation. The rates by which discussions about treatment impact on fertility and referrals to fertility specialists in women over the age of 40 , were exceedingly low. This is despite the fact that at least $16 \%$ of women in this age group expressed a desire for future pregnancy at the time of cancer diagnosis. This level of interest in future pregnancy among women over 40 years old is consistent with the increasingly common delay in child bearing, with 9.5 per 1000 births occurring in mothers age $40-44 .{ }^{16}$ We suspect that barriers to fertility-related care include inadequate physician awareness or support and insufficient institutional funding for appropriate fertility and sexual health specialist care.

This study has several strengths including its high participation rate of $90 \%$. The authors, however, acknowledge its limitations. Given the median time since cancer diagnosis of 2.4 years, patients could have been subject to recall bias when reporting the details of treatment planning discussions. A major strength of this study, however, is that it implemented a 1-year follow-up survey with duplicate questions from the original. This was completed by $54 \%$ of participants. The 1-year follow-up results demonstrate minimal variation compared with the original scores reported thus establishing consistency and validity of the data and limiting the possibility of recall bias.

This study was designed to minimize the possible influence of its conduct on how physicians would typically approach these topics or the content or duration of such reproductive and sexual health discussions with their patients. Physicians were removed from the recruitment and consent process, and all but the four physician investigators were blinded to the content of the study questionnaire. In addition, all eligible patients were either actively receiving cancer therapy or within 24 months of the completion of therapy at the time they completed the study questionnaire. Therefore the presence of the study investigators in the clinic could not change physician attitudes or approach to these discussions with the enrolling patients as their treatment planning and reproductive health conversations had already occurred.

Study participants in this single-institution study conducted at a large, academic medical center may not fully represent patient expectations, values or demographics in other geographic or more community-based locations. In addition, these women were recruited from 3 separate subspecialty oncology clinics as well as the general hematology and oncology clinic, and it is unknown whether subspecialty care and providers impact study results or if our conclusions would be fully applicable to a unified oncology practice staffed by general oncologists. However, this study included over 100 young women with a wide spectrum of malignant pathology. The needs, concerns, and opinions of these women that were elucidated from this study may be utilized to address and appropriately counsel an extensive population of young women facing any type of new cancer diagnosis.

In conclusion, one-third of pre-menopausal women with cancer are dissatisfied with the quality and length of oncologist discussions about the impact of cancer treatment on reproductive health. There is substantial room for improvement in communication and counseling of sexual health concerns, as well as the ability to provide resources and information for those women who demonstrate interest in fertility preservation. Further studies will be necessary to focus on the barriers to the oncologist's ability to provide such discussions and resources and for the development of interventions to overcome such barriers.

\section{Supplementary Material}

The Influence of Fertility and Sexual Health Discussions on Cancer Treatment Choice in Young Women. http://www.jcancer.org/v03p0217s1.pdf

\section{Acknowledgements}

The investigators would like to thank the Masonic Cancer Center's Health Survey Research Center for their expertise in the questionnaire design and formatting, and Michael Franklin of the University of Minnesota for his editorial support of this manuscript. 
The investigators also thank the Minnesota Medical Foundation for the Medical Student Research Grant funds that made this work possible.

\section{Competing Interests}

The authors have declared that no competing interest exists.

\section{References}

1 [Internet] Altekruse SF, Kosary CL, Krapcho M, et al. National Cancer Institute SEER Cancer Statistics Review based on the November 2009 SEER data submission, Revised in 2010. http://seer.cancer.gov/csr/ 1975_2007/

2 Partridge AH, Gelber S, Peppercorn J, Sampson E, Knudsen K, Laufer M, Rosenberg R, Przypyszny M, Rein A, Winer EP. Web-based survey of fertility issues in young women with breast cancer. J Clin Oncol. 2004; 22(20): 4174-83.

3 Thewes B, Meiser B, Taylor A, Phillips KA, Pendlebury S, Capp A, Dalley D, Goldstein D, Baber R, Friedlander ML. Fertility- and menopause-related information needs of younger women with a diagnosis of early breast cancer. J Clin Oncol. 2005; 23(22): 5155-65.

4 Nakayama K, Liu P, Detry M. Receiving information on fertility and menopause related treatment effects among women who undergo HSCT: changes in perceived importance over time. Biology of Blood and Marrow Transplantation. 2009; 11: 1465-1474.

5 Sonmezer M, Oktay K. Fertility preservation in female patients. Human Reprod Update. 2004; 10: 251-266.

6 Hickey M, Peate M, Saunders CM, Friedlander M. Breast cancer in young women and its impact on reproductive function. Human Rep Update. 2009; 15(3): 323-330.

7 Dizon D. Quality of Life after Breast Cancer: Survivorship and Sexuality. The Breast Journal. 2009; 15(5): 500-504.

8 Schover LR. Premature ovarian failure and its consequences: vasomotor symptoms, sexuality, and fertility. J Clin Oncol. 2008; 26: 753-8.

9 Leedham B, Ganz P. Psychosocial Concerns and Quality of Life in Breast Cancer Survivors. Cancer Investigation.1999; 17(5): 342-348.

10 Ganz PA, Rowland JH, Desmond K, et al: Life after breast cancer: understanding women's health-related quality of life and sexual functioning. J Clin Oncol. 1998; 16: 501-514.

11 Broeckel J, Thors C, Jacobsen P, Small M, Cox C. Sexual functioning in long-term breast cancer survivors treated with adjuvant chemotherapy. Breast Cancer Research and Treatment. 2002; 75: 241-248.

12 Wiggins DL, Wood R, Granai CO, Dizon DS. Sex, intimacy, and the gynecologic oncologist: survey results of the New England Association of Gynecologic Oncologists. J Psychosocial Oncol. 2007; 25(4): 61-70

13 Lee S, Shover L, Partridge A, Patrizio P, Hamish W, Hagerty K, Beck L, Brennan L, Oktay K. American Society of Clinical Oncology Recommendations on Fertility Preservation in Cancer Patients. J Clin Oncol. 2006; 24(18): 2917-2931.

14 Koczwara B. Addressing fertility needs of breast cancer patients: oncology perspective. Expert Rev Anticancer Ther. 2008; 8(8): 1323-30.

15 Schover LR, Rybicki LA, Martin BA, Bringelsen KA. Having children after cancer: a pilot survey of survivors' attitudes and experiences. Cancer. 1999; 86: 697-709.

16 Center for Disease Control. Births, Final data for 2007. National Vital Statistics Report 2010; 58(24): 1-88. 